\title{
Flexor Tendon Entrapment of the Index Finger After Monteggia Fracture in a Pediatric Patient
}

This article was published in the following Dove Press journal:

Orthopedic Research and Reviews

\author{
Akira Toga (iD) \\ Ayush Balaji (D) ${ }^{2}$ \\ Taisuke Matsumoto (iD) \\ Atsuki Fujimaru (iD) \\ Hideaki Murakami (iD) \\ Shojiro Katoh (iD) \\ 'Department of Orthopedic Surgery, \\ Edogawa Hospital, Tokyo, Japan; \\ ${ }^{2}$ Yokohama International School, \\ Yokohama, Japan
}

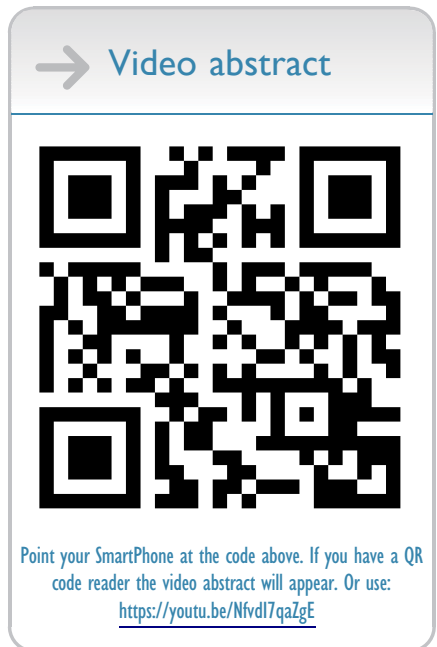

Correspondence: Akira Toga Department of Orthopedic Surgery,

Edogawa Hospital, 2-24-18 Higashikoiwa,

Edogawa-ku, Tokyo 160-8582, Japan

Tel +8I-3-3673-|22I

$\mathrm{Fax}+8 \mathrm{I}-3-3673-1229$

Email akira.toga@gmail.com
Abstract: The present case report describes a pediatric patient who presented with flexor digitorum profundus (FDP) entrapment after a forearm fracture. The patient was diagnosed with a Bado type I Monteggia fracture. The fracture was reduced using closed reduction under fluoroscopy followed by Kirschner's wire fixation. This case is unique because the FDP was found entrapped in the fracture site 2 weeks post-operation and was managed using conservative therapy. FDP entrapment is typically managed by surgical intervention, and there have been no previous reports of conservative management. The FDP was released using passive extension of the index finger under general anesthesia, and no irreversible damage to the tendon or muscle was found. This case report demonstrates the potential for conservative therapy in the management of FDP entrapment after forearm fractures.

Keywords: pediatric fracture, entrapment of FDP, conservative therapy, dynamic tenodesis effect, forearm fracture, rehabilitation

\section{Introduction}

The most common complications of Monteggia fractures are compartment syndrome and neurovascular damage. Entrapment or adhesion of the flexor digitorum profundus (FDP) at the fracture site is rare, and a standard treatment method has not yet been established. Currently, the most common treatment strategy is surgical intervention and release of the FDP tendon. In the present case report, we discuss a case of Monteggia fracture resulting in FDP entrapment that was treated conservatively.

\section{Case Report}

A 6-year-old right-handed girl who suffered a fall injury with an outstretched right hand arrived at our emergency room showing a fracture of the ulna shaft and a dislocated radial head. There was an open pinhole wound at the fracture site. Capillary refilling time was less than 2 seconds, and no circulatory disorders were found. The patient was diagnosed with a Monteggia fracture (Bado type I) by conventional radiography (Figure 1). She underwent closed reduction and internal fixation on the same day. Under general anesthesia, fixation was performed using a $1.2 \mathrm{~mm}$ Kirschner wire, and the area was lavaged. The radial head was treated using closed reduction and did not dislocate upon torsional stress with pronation and supination. The arm was fixed in a supine position until bone callus was visible on imaging using a forearm cast for approximately 3-4 weeks after the initial fracture, and an arm sling was used as immobilization. 


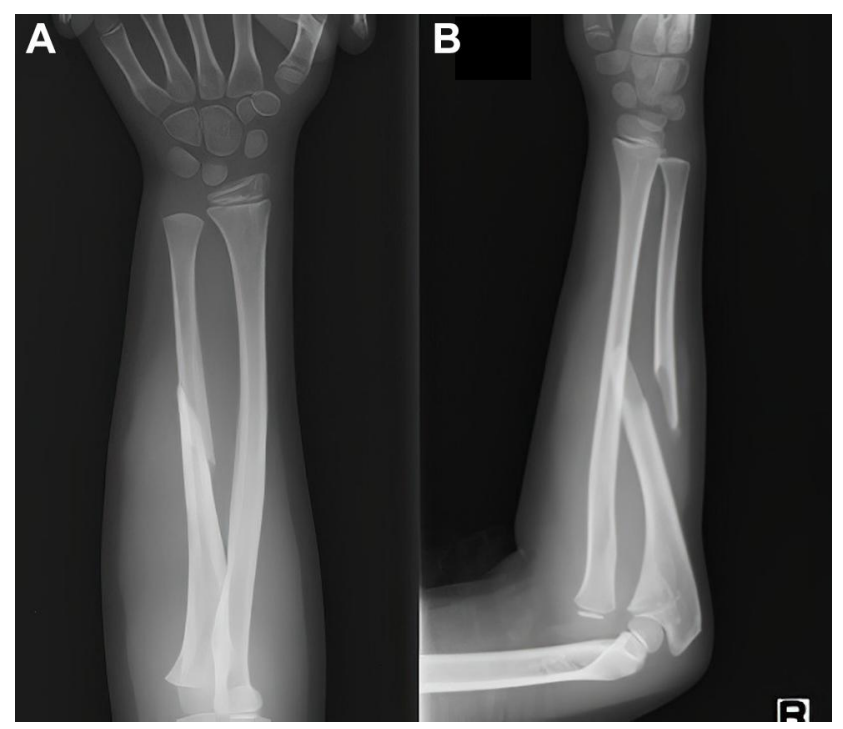

Figure I Preoperative anteroposterior and lateral radiographs ( $\mathbf{A}$ and $\mathbf{B}$ ) showing ulnar shaft fracture and dislocation of the radius head.

At the 2-week post-operation outpatient follow-up, the right index finger was unable to extend fully. Post-operative imaging on the 17 th day showed no dislocation of the radial head or abnormal callus formation (Figure 2). However, the active extension range of motion of the proximal interphalangeal (PIP) joint and distal interphalangeal (DIP) joint of the index finger was $-60^{\circ}$ and $-30^{\circ}$, respectively, at the $0^{\circ}$ wrist position (Figure 3). There was a limited flexion disorder of the 3 rd and 4 th digits. The extension disorder of the index finger was exacerbated when the wrist was under extension and relieved under flexion. Significant pain was observed during wrist extension, which prevented the patient from performing the action. The dynamic tenodesis effect was confirmed in the proximal part from the wrist joint. There were no vascular or neurological symptoms observed in the right arm, such as compartment syndrome or posterior interosseous nerve palsy. The clinical findings suggested that the right FDP may have become entrapped in the fracture site. Soft callus entrapment was excluded, owing to the timeline in which the entrapment occurred. Because the diagnosis occurred 2 weeks after the fracture, the likelihood of soft callus entrapment was considered low, and therefore direct entrapment was considered. An ultrasound (US) scan later confirmed direct partial entrapment of the FDP (Figure 4). We therefore made a diagnosis of partial entrapment of the FDP. Because the patient was unable to undergo a magnetic resonance imaging (MRI) scan, the main diagnostic techniques used were physical examination and US.

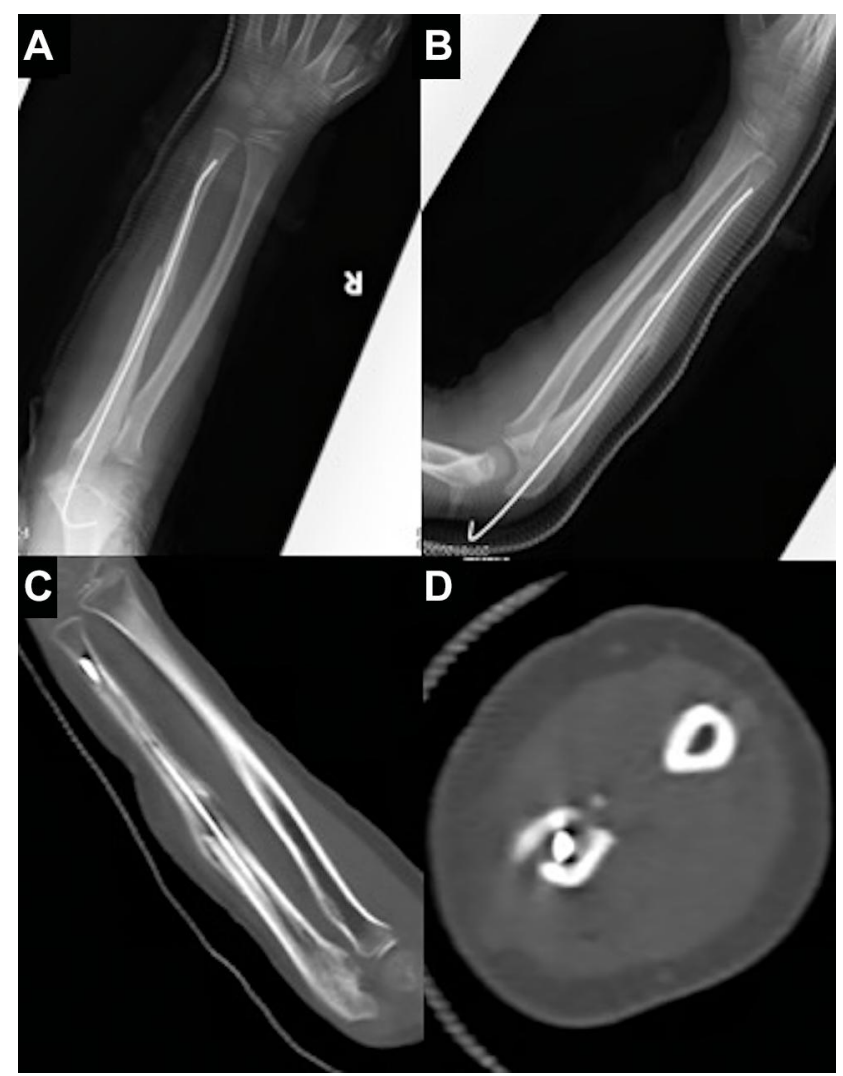

Figure 2 Postoperative anteroposterior and lateral radiographs ( $\mathbf{A}$ and $\mathbf{B}$ ); coronal and axial computed tomography (C and $\mathbf{D})$ on the 17th day showing no dislocation of the radial head or abnormal callus formation at the fracture site.

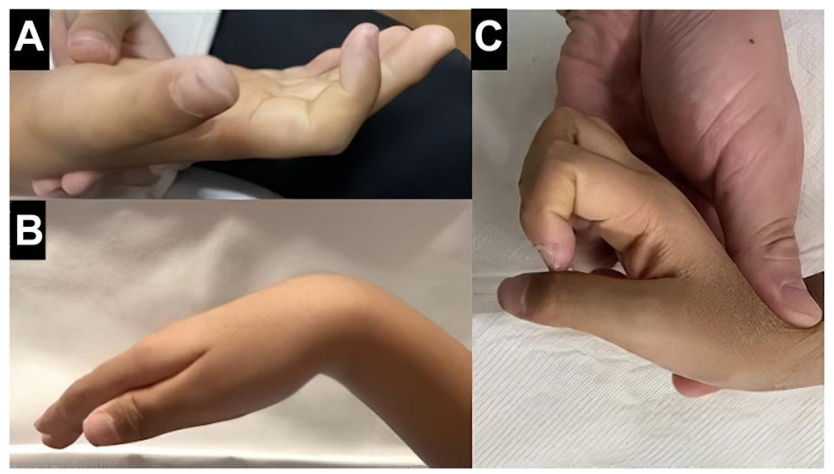

Figure 3 Clinical findings illustrating the extension lag of the index finger in the resting position $(\mathbf{A})$, which is less pronounced with wrist flexion $(\mathbf{B})$ and more pronounced with wrist extension (C). Extension of the index finger under wrist extension was conducted under general anesthesia due to pain $(\mathbf{C})$.

Three treatment options were planned, with joint mobilization as our first choice. If this was unsuccessful, open FDP release was the first backup. If the problem remained unresolved with both therapies, reproduction of the original fracture to release the entrapped FDP was planned. Passive joint mobilization using a delicate technique is necessary to prevent damage to the muscles or tendons. Care was taken 


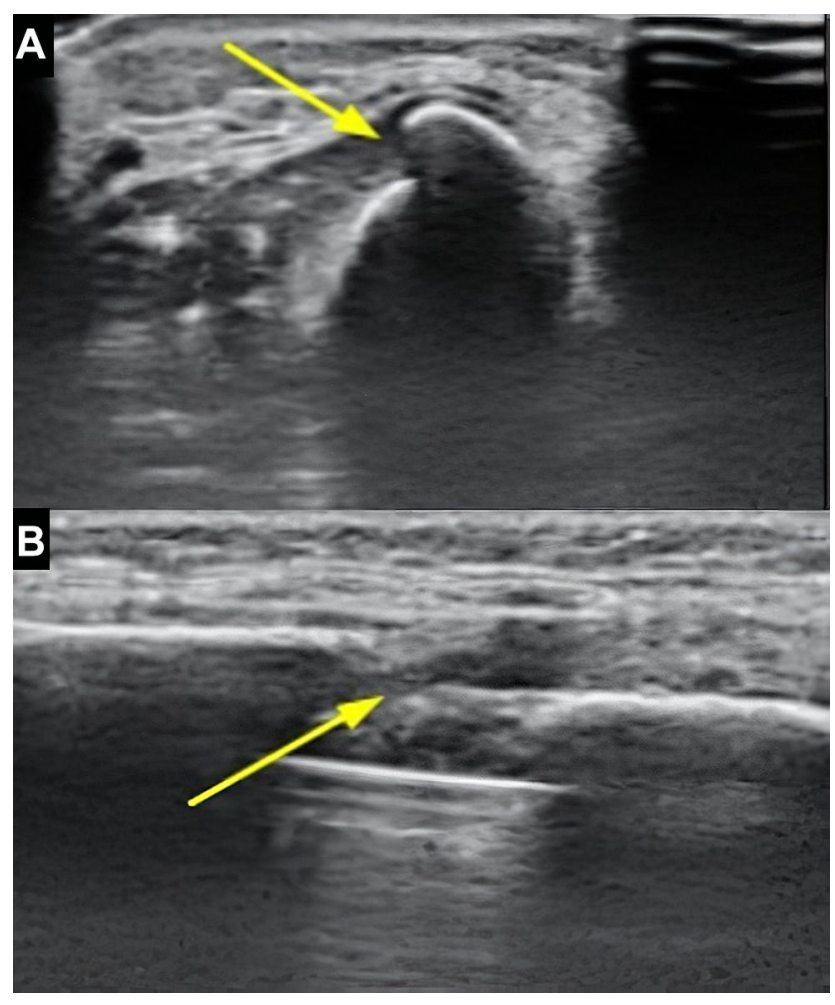

Figure 4 Longitudinal $(\mathbf{A})$ and transverse (B) sonogram of the ulnar shaft indicating partial FDP entrapment (yellow arrows) at the fracture site.

to avoid damage by tensile shear on the DIP joint. The patient was placed under general anesthesia, and with the right index finger gradually extended using passive motion, the PIP and DIP joints were extended to $0^{\circ}$ (Figure 5). A low amount of force was required for the extension of the fingers. FDP release was confirmed with physical examination of the finger. Immediately after surgery, weakness of the DIP joint was observed. However, the active extension range of motion of the PIP and DIP joints was $0^{\circ}$ at 1 month following joint mobilization, which was the same as the intact hand, while grip strength without pain reached half that of the intact joint. The PIP and DIP joints were able to achieve full range of motion when the wrist was extended without pain at approximately 6 months after treatment. The rehabilitation plan included swelling management, controlled passive motion, controlled active motion, and place and hold regimens to promote increased range of motion and restoration of strength. With extensive rehabilitation, the grip strength improved to the same level as that in intact hand. The flexion force of the DIP joint had also gradually recovered from approximately half that of the intact hand to the same level within 4 months. The patient had no problems with performing daily activities at the 8-month follow-up, and no other complications were observed

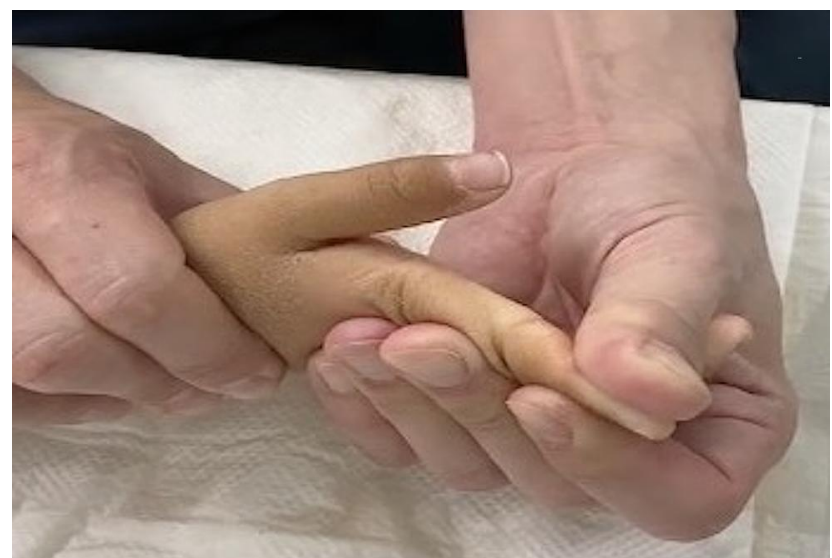

Figure 5 Passive joint mobilization of the right index finger enabling the PIP and DIP joints to extend to $0^{\circ}$.

(Figure 6). The patient was effectively able to use the affected hand and was able to perform intensive tasks without issue. At the follow-up visit, complete range of motion was confirmed and no deficits were visible. The postoperative care for the patient has lapsed after the 8 months checkup because the patient was determined not to have any deficits or pressing concerns.

\section{Discussion}

Finger extension disorders due to flexor tendon entrapment or adhesion as a complication of forearm fractures have rarely been reported in the literature. Twenty-one similar cases have been found to date. ${ }^{1}$ Surgical treatment was performed in all cases, and the fractured part was deployed openly to prevent the detachment of muscles and tendons. All subjects were aged between 5 and 17 years old. The majority of entrapments and adhesions occurred in the ulnar shaft. The period from injury to FDP release surgery ranged from 2 days to 16 years. ${ }^{1}$

A previous study suggested that two mechanisms underlie entrapment. ${ }^{2}$ In the first mechanism, the muscle belly becomes directly sandwiched between the fractured areas. This is referred to as acute entrapment and is the most common form. The second mechanism involves adhesion of the FDP to bone callus. This is referred to as chronic entrapment and is rarer due to the preventable nature of the condition. ${ }^{3}$ Treatment of acute entrapment should consist of re-manipulation with extension of the fingers, or open reduction and release of the muscle belly. ${ }^{2}$ For chronic entrapment, it is important to evaluate the dynamic tenodesis effect, and it is critical for the patient to undergo sufficient rehabilitation to restore full function of the tendon. 


\section{A}

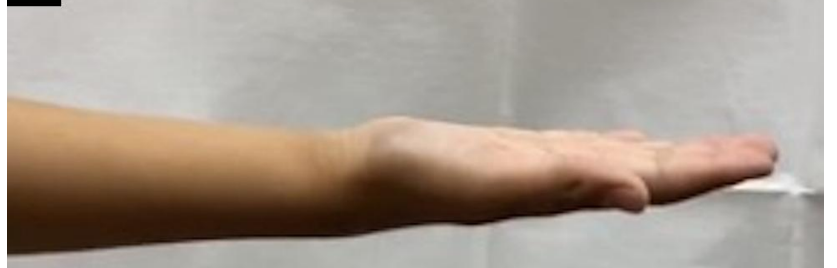

B
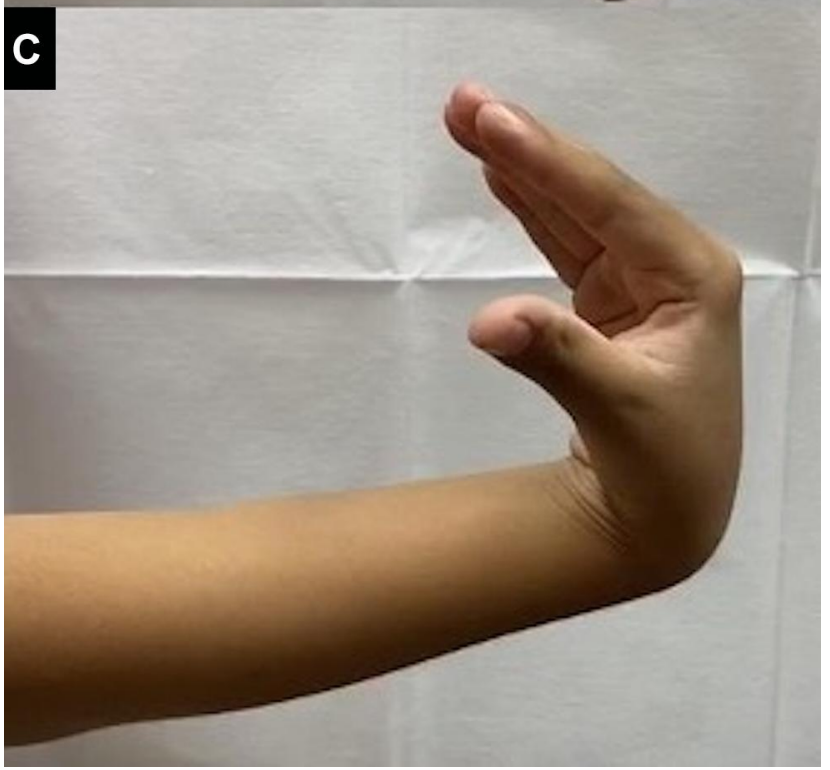

Figure 6 Range of motion 8 months post-FDP release. The PIP and DIP joints were able to have full range of motion without pain when the wrist position was neutral (A), extended (B), or flexed (C).

With regards to the diagnosis of FDP entrapment, it may be difficult to evaluate the dynamic tenodesis effect when casting is used due to restricted hand movement. In forearm fracture cases managed with conservative approaches, such as closed reduction and cast fixation, the diagnosis of FDP entrapment is often delayed. With conservative management, it is critical to evaluate the dynamic tenodesis effect and check for pain or stiffness during wrist extension. Page et al also reported that FDP entrapment commonly allows for increased finger extension and decreased pain when the wrist is flexed or neutral compared to when the wrist is extended. ${ }^{1}$ In addition to clinical findings, computed tomography $(\mathrm{CT})$, magnetic resonance imaging (MRI), and ultrasound sonography (US) are also effective diagnostic tools. ${ }^{4}$ Preoperative ultrasound may serve as an alternative to MRI, especially in pediatric patients because it is non-invasive, cost-effective, and less time-consuming.

Surgical interventions represent the most common treatment strategy for FDP entrapment to date, but several reports have suggested that conservative treatments may be worth exploring. ${ }^{5}$ Indeed, a conservative approach may be worth considering as a primary treatment method due to its ability to promote recovery through rehabilitation. Our literature review revealed no instances of muscle or tendon damage with flexor tendon rehabilitation. One important factor to consider when determining a treatment plan is the location of the entrapment site. The FDP muscle-tendon junction is located in the distal $1 / 3$ of the ulna, and the muscle belly of the FDP is located approximately in the middle $1 / 3$ of the proximal ulna. Therefore, conservative treatment may be considered in fracture cases involving the proximal $1 / 3$ because it is proximal to the muscle-tendon junction, thus reducing the risk of tendon tear. When entrapment occurs in the distal $1 / 3$, surgical treatment should be considered. ${ }^{6-8}$ Another factor when considering the choice of treatment is the degree of entrapment, which should be evaluated by traditional diagnostic methods along with imaging. Complete and partial entrapment can be differentiated using ultrasound sonography, CT, or MRI. Partial entrapment of the FDP could benefit from conservative management and carries a lower risk of tendon damage compared with total entrapment. It is important to prepare for open release of the FDP tendon when there is suspected muscle or tendon damage to prevent further complications. The secondary effects of FDP entrapment can be mitigated by early diagnosis and intervention; therefore, guidelines are needed to support the diagnosis of FDP entrapment in forearm fractures.

\section{Ethical Statement}

The study was approved by the review board at Edogawa Hospital. 


\section{Informed Consent}

Informed consent was obtained from the patient and her parents for publication.

\section{Acknowledgments}

The authors would like to thank Medical Journal Editors (MJE) for assistance in the editing of our manuscript.

\section{Funding}

The authors received no financial support for the research, authorship, and/or publication of this article.

\section{Disclosure}

The authors declare no potential conflicts of interest.

\section{References}

1. Page F, Yacomel T, Ting J, et al. Tenodesis effect mimicked by flexor tendon entrapment in a paediatric midshaft forearm fracture: a case report and review of the literature. SN Compr Clin Med. 2020;2 (3):365-372. doi:10.1007/s42399-020-00230-2
2. Deeney VF, Kaye JJ, Geary SP, Cole WG. Pseudo-Volkmann's contracture due to tethering of flexor digitorum profundus to fractures of the ulna in children. $J$ Pediatr Orthop. 1998;18(4):437-440. doi:10.1097/01241398-199807000-00005

3. Hendel D. Prevention of chronic entrapment of the ring finger flexor profundus muscle in ulnar shaft fractures in children - a case report. Acta Orthop. 2004;75(4):1. doi:10.1080/759369199

4. Pandey T, Al Kandari SR, Al Shammari SA. Sonographic diagnosis of the entrapment of the flexor digitorum profundus tendon complicating a fracture of the index finger. J Clin Ultrasound. 2008;36 (6):371-373. doi:10.1002/jcu.20460

5. Littlefield WG, Hastings H 2nd, Strickland JW. Adhesions between muscle and bone after forearm fracture mimicking mild Volkmann's ischemic contracture. J Hand Surg Am. 1992;17(4):691-693. doi:10.1016/0363-5023(92)90318-J

6. Fernandez ML, Segal LS. Entrapment of the flexor digitorum profundus following paediatric forearm fractures. Acta Orthop Belg. 2007;73(6):780-784

7. Watson PA, Blair W. Entrapment of the index flexor digitorum profundus tendon after fracture of both forearm bones in a child. Iowa Orthop J. 1999;19:127-128.

8. Brogan K, Nicol S. Flexor digitorum profundus entrapment in paediatric forearm fractures. J Surg Case Rep. 2014;2014(5):rju038. doi:10.1093/jscr/rju038

\section{Publish your work in this journal}

Orthopedic Research and Reviews is an international, peer-reviewed, open access journal that focusing on the patho-physiology of the musculoskeletal system, trauma, surgery and other corrective interventions to restore mobility and function. Advances in new technologies, materials, techniques and pharmacological agents are particularly welcome. The manuscript management system is completely online and includes a very quick and fair peer-review system, which is all easy to use. Visit http://www.dovepress.com/testimonials.php to read real quotes from published authors. 